\title{
MUERTE Y PSICOTERAPIA
}

\author{
Hector Fernández-Alvarez' \\ Universidad de Belgrano, Buenos Aires
}

\begin{abstract}
RESUMEN
La psicoterapia trata de la experiencia del dolor y del sufrimiento que, en determinadas circunstancias, aquejan al ser humano. Se trata de formas del padecimiento en que se cumplen dos premisas básicas: el dolor no tiene sentido para quien 10 padece, y el guión que hasta entonces orientaba su vida se interrumpe bruscamente. Una psicoterapia exitosa será aquella que permita al individuo continuar con el cumplimiento de su guión personal, facilitando los cambios y las reparaciones necesarias para ello. Por lo que se refiere a la muerte, hay muchas que se producen en el transcurso del proceso de la psicoterapia, aunque no siempre se hable de ellas. Existen ciertas situaciones en las que la psicoterapia trabaja sobre focos tan acotados y definidos, que es posible recorrer la totalidad del proceso psicoterapéutico sin que tengamos oportunidad de encontrarnos con la muerte. ¿Cuáles son pues las muertes de las que se habla en la psicoterapia?. Podemos distinguir las muertes que forman parte del texto del paciente, de aquellas otras que corresponden al universo del terapeuta. En este trabajo, nos ocupamos de las primeras, que pueden agruparse en tres grandes conjuntos: 1) las muertes ya ocurridas, 2) las muertes que ocurren durante la terapia y, 3) la experiencia de la propia muerte.
\end{abstract}

PALABRAS CLAVE: Muerte, psicoterapia, enfermedad y psicoterapia.

\begin{abstract}
Psychotherapy is directly engaged with the pain and suffering when under some circumstances it hits to the human being. In these experiences there are two basic premises: the pain seems to have no sense for the person who suffers and the script that has been directing his/her life is suddenly interrupted. A psychotherapy can be considered successful anytime it allows the person to go on with his personal script, whether it facilitates the changes which are necessaries to go on. With regard to death, there are many deaths which occur in a psychotherapy process but not always they are fully expressed. There are certain situations in psychotherapy where the focus is so narrow that we can cover a whole psychotherapy experience totally without having the opportunity of mentioning death. Which are the deaths that are talked in psychotherapy?. We can distinguish those that are part of the text of the patient from those that correspond to the universe of the therapist. In this work, we will taik about the first ones, that can be grouped in 3 major set: 1) deaths already occurred, 2) deaths that occur during psychotherapy, and 3) the experience of ones own death.
\end{abstract}

KEY WORDS: Death; psychotherapy; death and psychotherapy; illness and psychoterapy.

' Correspondencia: H. Fernández Alvarez, Aiglé. C.C. 135. Suc. 26. 1426- Buenos Aires. Argentina. 


\section{INTRODUCCIÓN}

¿Quién querria llevar tan duras cargas, gemir y sudar bajo el peso de una vida afanosa, si no fuera por el temor de un algo después de la muerte, esa ignorada región cuyos confines no vuelve a traspasar viajero alguno, temor que confunde nuestra voluntad y nos impulsa a soportar aquellos males que nos afligen, antes que lanzarnos a otros que desconocemos? Asi la conciencia hace de todos nosotros unos cobardes; $y$ asi los primitivos matices de la resolución desmayan bajo los pálidos toques del pensamiento, y las empresas de mayores alientos e importancia, por esa consideración, tuercen su curso y dejan de tener nombre de acción.

Hamlet, acto III.

La psicoterapia tiene que ver con la experiencia del dolor y el sufrimiento que bajo determinadas condiciones y circunstancias aquejan al ser humano. Se trata de formas del padecimiento en que se cumplen dos premisas básicas: el dolor no tiene sentido para quien lo padece, y el guión que orienta su vida se enfrenta a obstáculos que amenazan la continuidad del curso esperable hasta entonces, o se ha quebrado y está impedido de seguir desplegándose. Una psicoterapia exitosa habrá de facilitar los cambios y las reparaciones necesarias que permitan continuar con el cumplimiento de un guión personal.

Como sabemos, el punto final de ese curso es la muerte. Jaspers (1967) examinó su condición de situación límite. Su carácter ineludible le otorga tal certidumbre que se nos presenta como la única verdad absoluta con que se cruza la experiencia humana. Levinas (1994) sentenció notablemente que "la muerte es el demumbamiento de la apariencia".

Conjeturar sobre el espacio que se abre más allá de ese límite que es el morir, ha sido una de las preocupaciones principales de los seres humanos a través de los tiempos. Pensando en ello Manrique supo decir que "este mundo es el camino para el otro, que es morada sin pesar". Mostraba en ello su firme convicción de que solo alli podíamos encontrar verdadero alivio y descanso para las duras condiciones de nuestra vida terrenal. Para quienes, como él, sostienen una creencia religiosa, la muerte es una puerta que nos introduce en la gran posibilidad. Incluso se debe aspirar a ella, como se deduce de las palabras de San Agustín: "Por tanto, para poder sumergirse sin dificultad, con todo su ser, en la plenitud de la verdad, el alma anhela como don supremo aquella completa redención y liberación del cuerpo, la muerte". O de las de Calvino: "A los hijos de Dios no sólo les es licito desear para si la muerte, sino que tienen además el deber de hacerlo, pues muestran la verdadera ratificación de su fe tan sollo cuando anhelan dejar este mundo".

Para Hamlet, situado desde una perspectiva muy diferente, experimentamos temor ante su umbral, inseguros frente a la infinita incertidumbre de lo que a partir de alli ocurrirá. La historia del pensamiento humano, de la filosofia, del arte, de la cultura en general, puede entenderse como una larga reflexión sobre este pasaje que separa la vida de la muerte. A través de los siglos, cada grupo social, cada conglomerado humano, tuvo algo que decir sobre ello. La evolución del espíritu humano puede leerse como la larga peregrinación en torno a ese eje configurador de la experiencia, como el incesante trabajo de reinterpretación sobre lo inefable 1 .

La tarea psicoterapéutica está situada completamente en la matriz de esta escena que nos delimita de manera permanente. Yalom (1984) presentó uno de los más completos estudios sobre su naturaleza y sus implicancias para la psicopatología y la psicoterapia. Podemos ob servar dicha escena desde tres ángulos: a) como modos de interpretar determinados acontecimientos vitales, b) como formas concretas de manifestación y, c) como móvil de una actividad reflexiva. Considerando el primero de

\footnotetext{
${ }^{1}$ Existe una abundante y heterogénea bibliografla sobre el tema. Aries (1983) y Thomas (1983) son algunos de los textos más abarcadores. Hertz (1990) presenta un interesante estudio sobre la representación colectiva de la muerte.
} 
estos aspectos, la muerte aparece en la psicoterapia desde ópticas muy dispares: en la preocupación de los pacientes cuando se ven enfrentados a determinados síntomas, en las observaciones que tanto pacientes como terapeutas realizan sobre algunos acontecimientos vitales significativos o como parte del núcleo teórico de cada modelo de trabajo. En el segundo sentido, la muerte se actualiza concretamente en aquellos episodios que pueden sobrevenir durante el transcurso de la terapia, o manifestarse en la memoria, como referencias de una historia que reaparece de manera incesante. Desde este ángulo, la inevitabilidad futura de la muerte se abalanza, en el espacio terapéutico, sobre cada momento presente. En último término, su presencia se patentiza, con frecuencia, por medio de diferentes interrogantes éticos relacionados con el sentido de lo que cada ser humano emprende dia a día. Spinoza afirmó en la Proposición LVXII de su Etica: "El hombre libre en ninguna cosa piensa menos que en la muerte, y su sabiduria no es una meditación de la muerte, sino de la vida".

En otras palabras, muchas son las muertes de las que se habla en la psicoterapia, muchas son las muertes que se evitan o se enfrentan en el curso de una psicoterapia. ¿Acaso se habla siempre de ella?. No siempre; existen situaciones que permiten trabajar sobre focos $\tan$ acotados, tan definidos, que es posible recorrer la totalidad de una experiencia psicoterapéutica (que entonces no será ni muy larga ni muy profunda) sin que tengamos oportunidad de encontrarnos con ella. Pero aún en esos casos, estará recostada en el fondo del escenario, se encuentra siempre colocada como un telón ineludible que enmarca toda la trama sobre la que se representa el drama de cada paciente.

¿Cuáles son, en sintesis, las muertes de las que se habla en la psicoterapia?. En su transcurso, se pueden distinguir las muertes que forman parte del texto del paciente, de aquellas otras que corresponden al universo del terapeuta. En este trabajo, nos ocupamos de las primeras, que pueden agruparse en tres grandes conjuntos: 1) las muertes ya ocurridas, 2) las muertes que ocurren durante la terapia y 3) la experiencia de la propia muerte. Luego de una breve descripción de cada una de estas modalidades, exponemos algunos principios aplicables en la labor terapéutica.

\section{LAS MUERTES YA OCURRIDAS}

¿Cuáles son estas muertes?. Son las muertes de los otros, de aquellos familiares, amigos y conocidos que desaparecieron en el transcurso de la vida de la persona que nos consulta. Desde el momento en que mantenemos una primera entrevista, en el instante en que confeccionamos un primer mapa de lo que afecta a la persona que nos consulta, elaboramos un primer bosquejo de lo que le ocurre y de las circunstancias que han conformado su experiencia hasta ese momento. Ya en ese momento inicial, solemos oir hablar de un padre o de una madre muertos; también podemos oir mencionar, quizás con mayor seguridad, que uno o varios abuelos han perecido; y aunque no tan frecuentemente, podemos oir hablar de otras personas muertas en variadas circunstancias. Pues son innumerables las formas de morir.

Esta aparición temprana del tema tiene, a veces, el carácter de un mero registro necrológico, una suerte de burocracia de la clínica que sirve para organizar los datos epidemiológicos, o para estructurar una ficha de admisión. (Padre muerto hace tantos años en tales circunstancias, madre muerta hace tantos meses como resultado de una larga enfermedad, etc.). Sin embargo, no siempre es así. A veces ese dato, esa temprana información de una muerte familiar adquiere, desde un comienzo, una extraordinaria importancia en relación a la consulta que hace el paciente: la depresión que arrastra parece relacionarse con ese hecho, o la imposibilidad de tejer nuevas propuestas para su vida parecen estar vinculadas con la perduración de un duelo que no puede elaborarse. Nuestra relación con el paciente puede, en esos casos, quedar ligada desde el inicio de la terapia a esa muerte ocurrida tiempo atrás y que seguirá sobrevolando el espacio del tratamiento por un periodo prolongado. Incluso, a veces, el destino de la terapia en su conjunto estará condicionado a la manera en que se pueda resolver esta circunstancia.

Insistimos con esta palabra, circunstancias, porque no se trata sólo de morir. En los seres humanos, la muerte tiene mucho que ver con 
la forma en que ocurre, con el momento en que sobreviene, con el estilo en que se produce. No es lo mismo morir de pronto, súbitamente, que morir en forma progresiva, gradual; no es igual una muerte precedida de grandes dolores físicos que otra directa, inmediata, producto de un accidente o de un asesinato; no es lo mismo morir a una cierta edad que una década más tarde y asi siguiendo. Sobre todo, no es lo mismo morir rodeado del afecto y el cuidado de los otros que morir en soledad, olvidado, y sin tener alguien cerca que pueda acompañarnos en ese tremendo instante. Una detallada descripción sobre las formas de morir se presenta en Thomas (1983).

Cuando oímos hablar tempranamente de las muertes ocurridas en torno a nuestros pacientes, los detalles parecen adquirir una extraordinaria importancia, pues orientan el examen destinado a conocer las cruciales experiencias vitales que debió atravesar la persona en su vida hasta el instante en que se produce nuestro encuentro. Carlos (25 años), se presentó diciendo que necesitaba nuestra ayuda porque se sentía muy angustiado y completamente confundido respecto a qué hacer con su vida. Además nos habló, en el curso de nuestro primer encuentro, que su madre había fallecido unos diez años atrás y que su padre se había suicidado dos años antes de nuestra entrevista. Estábamos recién tomando contacto con él, estábamos estableciendo una primera aproximación a su problema, a su dolor, cuando de pronto, estábamos siendo testigos de ese suicidio ocurrido en una madrugada, mientras él había salido de su casa. El paciente nos introduce en la escena, nos invita a acompañarlo: alli estamos, caminando con él, desprevenidamente, en dirección a su casa, volviendo de una salida nocturna y de pronto, nos muestra su visión de una persona caida junto a un árbol, mientras otra se acerca, alborotada; a medida que ésta se aproxima reconoce en su figura a una tia y en su mirada de horror comprende que está mirando en el cuerpo del caído el cuerpo de su propio padre. Nos acercamos, más cerca aún, se da cuenta que está sangrando...

Esta inundación de lo vivencial en el campo psicoterapéutico no es privativa de la muerte; los pacientes suelen invitarnos o empujar- nos directamente hacia escenas de violencia y de un grado de crudeza que ponen a prueba permanentemente la vocación para ejercer este oficio de terapeuta. A menudo, algunos profesionales descubren alli su verdadero límite para el desempeño en una tarea que obliga, con frecuencia, a ser testigo de actos cuyo tremendismo pone en evidencia que las noticias publicadas por los medios no constituyen historias exageradas, sino meros ejemplos de acontecimientos que forman la trama de la vida cotidiana.

¿Qué lugar ocuparán las muertes ya ocurridas en el curso de una psicoterapia?. A veces estarán suficientemente bien elaboradas y pasarán a formar parte del registro que haremos de la historia del paciente. Pero en muchas ocasiones, serán el lugar que conecta con una herida todavia abierta y que intentaremos ayudar a suturar.

Las muertes ya ocurridas pertenecen, pues, a dos campos. Uno, mediato, al que accedemos por medio del registro de una sucesión histórica que nos permite configurar algunos de los hitos fundamentales de la vida del paciente. Otro, inmediato, que suele organizarse como un atributo fundamental del padecimiento que ha traido al paciente a la consulta. Son las muertes de los familiares que han sumido al paciente en un estado depresivo del que no puede reponerse, las desapariciones de aquellos (padres, parejas, hermanos), que han dejado un vacio que no puede llenarse.

¿Cuáles son las figuras de estos muertos que ocupan el centro del tratamiento con mayor frecuencia?. Hay tres prototipos fundamentales: 1) las que están sostenidas por un sentimiento de culpabilidad que impide terminar de realizar un duelo, y por ende, someten al paciente a una vivencia de intranquilidad perpetua; 2) las que están recubiertas con recuerdos tan fuertemente negativos que obstaculizan las posibilidades del paciente para avanzar en la constitución de su identidad personal; 3) las que designan un espacio vacío que debe ser cubierto y definen, por lo tanto, la existencia de un fantasma que llama al paciente a un convite tenebroso.

Hamlet es un paradigma que sintetiza, en alguna medida, todas esas formas. Un crimen ha sido cometido y la persona siente que alguna 
responsabilidad le cabe en ello. Puede ser por alguna acción cometida, o bien por omisión. Algo que la persona hizo o dejó de hacer tiene que ver con la muerte de otro que lo increpa desde el reino de los muertos y le muestra siempre viva la llaga de la culpa. Con frecuencia, estas situaciones se vinculan con algo que no se dijo, algo que debería haberse dicho y no se llegó a comunicar, en especial un sentimiento conflictivo. Veamos cada una de estas figuras y qué podemos hacer con ellas.

\section{Los muertos que despiertan sentimientos de culpabilidad}

La madre de Pedro falleció hace 3 años, en un instituto geriátrico, donde había sido internada por su hijo cuando comenzó a mostrar los primeros signos de declinación física. Pedro (41 años), siente, todavía hoy, culpa por ello, $e$ interpreta que la rapidez con que sobrevino su muerte es el resultado del apresuramiento con que tomó la decisión de internarla. Piensa en ello como muestra de su desapego 0 , al menos, como señal de su falta de coraje para enfrentar el envejecimiento de su madre. Debilitado por estos sentimientos que afectan su valoración personal, se siente inseguro y confundido en el manejo de sus relaciones primarias.

Los muertos que nos generan sentimientos de culpabilidad son aquéllos ante quienes pensamos haber hecho cosas que propiciaron ese desenlace o no haber hecho lo suficiente por evitar su muerte. $O$, simplemente, son aquellas personas muertas con las que nos liga la sensación de haberles fallado, o de no haberles transmitido la importancia que tenian para nosotros o la gratitud que le debiamos. Hay especialmente en estos casos, casi siempre, la falta de un intento de explicación o justificación respecto del modo en que se desarrollaron los hechos. Por eso mismo, muchas personas se sienten mejor cuando pueden "despedirse" de un moribundo, 10 que significa poder transmitirle ciertos sentimientos ocultos, a veces cariñosos, pero otras veces hostiles, que se supone deberian manifestarse para lograr tranquilidad.

En estos casos, la labor de la psicoterapia puede seguir dos rumbos: uno, consiste en ayudar al paciente a revisar sus esquemas de clasificación, con el fin de examinar sus posibilidades para emprender una nueva manera de ordenación de los datos que le permita construir una historia más equilibrada de los intercambios en esa relación. En situaciones más severas o resistentes, deberá ayudarse al paciente a propiciar acciones reparatorias, capaces de reequilibrar los esquemas centrales de valoración personal.

\section{Los muertos cargados negativamente}

Experiencias de dificil elaboración de una muerte pueden ocurrir aunque la persona muerta no despierte sentimientos de culpabilidad en el paciente. Silvina (25 años), habia perdido a su padre a los 12 años y su muerte habia significado un alivio en la familia. Todo lo que ella recordaba es que su conducta producia dolor y sufrimiento para su madre, así como para ella y sus hermanas. Por lo tanto, su muerte había sido seguida por una suerte de liberación. El paso de los años había contribuído a verificar ese sentimiento y perfeccionar las imágenes negativas de aquel hombre cruel que sólo les había aportado sufrimiento a sus vidas. A medida que la relación terapéutica comenzó a progresar, se fue haciendo evidente que semejante recuerdo de su padre operaba como un obstáculo muy fuerte para que ella pudiera concretar ciertos proyectos que, por otra parte, ansiaba intensamente. Un lugar tan negativo de la figura del padre muerto contribuia a una, desvalorización muy generalizada de los hombres, lo que conspiraba haciendo que todo proyecto de transitividad se viera permanentemente dificultado.

El trabajo terapéutico se orientó hacia una gradual reclasificación de las estructuras de significado que contenian los recuerdos de la vida familiar. Poco a poco, la paciente pudo ir descubriendo cómo había operado, a través de los años, el modo materno de construcción en relación con la figura de su padre. Silvina comenzó a observar una equivalencia total entre lo que ella pensaba de su padre y la construcción que su madre habia hecho de él como resultado de la conflictiva relación de pareja sostenida a través de muchos años. Lentamente, la paciente pudo ir modificando esa cons- 
trucción. Lo llevó a cabo de manera muy laboriosa, por medio de un trabajo de investigación en varios planos, que incluyó el rastreo de diversos materiales, diálogos con otros familiares y el regreso a su barrio de origen donde pudo escuchar testimonios de vecinos que guardaban una imagen muy positiva de su padre. La imagen de su padre no llegó nunca a ser muy positiva, pero dejó de ser frontalmente negativa. Tiempo después inició su primera experiencia sólida de relación afectiva con un hombre en quien veía diversas cualidades positivas y con quien tuvo poco después dos hijas a las que quería entrañablemente.

\section{Los muertos fantasmas}

A veces el paciente que nos consulta cree que debe responder al mandato de ocupar el lugar de un muerto. Esto puede asumir dos formas. Una, relacionada con la sucesión generacional, implica un camino franco de exigencias identificatorias, cuyos alcances pueden ser muy variados, dependiendo de la positividad o negatividad de la carga y de la magnitud de la misma. Los pacientes pueden verse, en esas circunstancias, teniendo que continuar la tarea o el proyecto de una figura paterna 0,10 que también es muy frecuente, una figura de la generación anterior, un abuelo o abuela que constituyen núcleos del mito familiar. El paciente pudo haber conocido o no a esa persona, pero de todas maneras se siente encarnándolo en sus actos y en sus proyectos. En general, estas figuras resultan especialmente conflictivas cuando están saturadas de un modo negativo en algún aspecto significativo. En alguna medida, eso constituye una variante de la situación anterior.

Otras veces, ese lugar es un lugar que ha quedado vacio en la fratria y el paciente es llamado a ocuparlo. El paciente es el muerto renacido y esa resurrección lo obliga a un diálogo con el muerto que oficia de fantasma en su vida cotidiana. El caso más habitual es el de un hermano muerto que ha dejado una marca muy importante en el universo afectivo de los padres. El paciente que nos consulta nos habla de aquél a quien vino a reencarnar, de sus temores frente a ello y de la exigencia de tener que ser otro que no es él mismo en todo momento. Debe enfrentar esos dos dramas: el temor de que le ocurra lo mismo que a su hermano, el temor de que el fantasma lo venga a buscar, celoso de que él esté viviendo y deseoso de llevarlo con él; y el peso de tener que cubrir las expectativas que estaban depositadas en aquél, obligándolo a cumplir un proyecto permanentemente ajeno.

Julián era un joven ingeniero, exitoso profesional y socialmente, que requirió ayuda a raíz de los efectos colaterales de una reacción convulsiva aguda, padecida a los 32 años. Estudios neurológicos posteriores detectaron la presencia de una mancha en el cerebro que dio lugar a diferentes interpretaciones diagnósticas. La más consistente, definió la imagen como un angioma, cuya única solución era, en principio, quirúrgica. Sin embargo, la complejidad y el riesgo de la operación dificultaban tomar una decisión, pues la evolución del problema también resultaba incierta. El perjuicio más inmediato resultaba la exposición a crisis convulsivas periódicas que se presentaron, efectivamente, en el momento inmediatamente posterior al ataque inicial. Julián habia nacido con posterioridad a la muerte de una hermana mayor, muerte de la cual su madre no se repuso totalmente, nunca más. La consecuencia más evidente resultó una larvada depresión que hacia periódicas manifestaciones.

El trabajo terapéutico se dirigió a una reestructuración cognitiva de aquellos esquemas centrales de Julián que guardaban un núcleo hiponcondriaco, vinculado con la conflictiva posición que ocupaba en el sistema familiar. En la medida que pudo afirmar su guión personal, pudo concebir su vida como algo propio, desligándola del deseo materno de compensar la pérdida de su hermana. En la misma dirección, pudo evaluar sus éxitos como algo vinculado a sus actividades, desconectándolo de otras tantas compensaciones. En la medida en que su vida le fue perteneciendo, su angustia disminuyó y las convulsiones comenzaron a espaciarse. Continuó con estudios neurológicos de seguimiento (incluyendo la consulta a una eminencia mundial que residia en otro pais), sin que fuera necesario proceder a la intervención quirúrgica. Esta situación se mantiene en la actualidad, varios años después de 
haber terminado su psicoterapia y a más de 15 años de la crisis inicial.

\section{LAS MUERTES QUE OCURREN DURANTE LA TERAPIA}

Hay otras muertes de las que se habla durante la terapia: son las de aquellos familiares, amigos y conocidos que ocurren durante el proceso de la terapia. Cuando los psicoterapeutas aceptan trabajar con metas abiertas, acordando con el paciente un objetivo que implica explorar construcciones primarias de su representación del mundo, se preparan para establecer una relación interpersonal prolongada. En esas condiciones, los terapeutas aumentan su grado de exposición y se ven convocados, con frecuencia, a la necesidad de acompañar al paciente en importantes y dramáticos acontecimientos de su vida.

Ejemplos de ello se producen cuando nos encontramos trabajando con un paciente quien, en algún momento de su tratamiento nos informa que un familiar (o amigo o conocido) ha enfermado gravemente. De alli en más, nos tocará asistir a su deterioro progresivo (acelerado o no) hasta llegar a su muerte. Otras veces nos impacta directamente la noticia de que alguien ha muerto. ¿Qué es lo que se espera del terapeuta en esas circunstancias?. Soslayemos la solución obvia de "proponer una acción que dependa de cada caso". ¿Qué lugar deberá darse a esos acontecimientos en el contexto de una tarea que tiene otros fines?. ¿Es esperable, por ejemplo, que un terapeuta asista al velatorio de un familiar 0 de un allegado al paciente? ¿Cómo determinar el grado de significación que puede tener para el tratamiento la conducta que el terapeuta adopte en esas circunstancias?. ¿Cómo deberá conectarse con el rito de esa muerte que afecta al paciente?

Aunque sabemos que en cada caso lo más útil será algo diferente y que, en buena medida, lo más acertado tendrá que ver no sólo con los acontecimientos que afronta el paciente sino además con el estilo personal del terapeuta, sabemos también que el paciente estará evaluando el modo en que el terapeuta oficia en ese pasaje que él experimenta, la manera en que se sitúa frente a ello. Como en otras situaciones límites, el paciente esperará que su terapeuta lo contenga frente al excesivo dolor que padece, o supondrá que el terapeuta estará capacitado para explicarle por qué ha ocurrido lo que para él es inexplicable o bien, en una situación extrema, le exigirá que rinda cuentas de por qué no pudo evitar que el paciente pase por una situación tan dolorosa.

Lo común a todas estas expectativas es la agudeza con que ponen a prueba la capacidad del terapeuta para regular la omnipotencia. Ya que, en todas ellas, el terapeuta deberá mostrar que, a pesar de su incapacidad para diluir ciertas escenas de sufrimiento, puede conservar su rol en relación con los objetivos trazados. Este hecho de tener que afrontar ante el paciente la conciencia de sus límites es un punto crítico de muchos tratamientos, y un momento crucial que pone en juego la habilidad de todo terapeuta.

Las muertes ocurridas durante el tratamiento pertenecen a dos categorías: unas son muertes "lógicas"; otras son "absurdas". En las primeras, el paciente tenía alguna expectativa de que podrian ocurrir, y la forma en que se producen no constituye una factor sorpresivo. En esos casos, los sentimientos acompañantes suelen ser calmos, aunque puedan variar en intensidad, desde experiencias muy dolorosas hasta otras muy desligadas. El paciente suele enfrentar esta experiencia sin la sensación de padecer una ruptura en el cumplimiento de su guión personal. Las muertes absurdas, por el contrario, sobrevienen en un momento impensado o se presentan de un modo particularmente cruento. El paciente relata una vivencia en extremo dolorosa y tiene sensaciones de un quiebre que lo sustrae de la tarea en la que estaba concentrado, para pasar a ocupar (transitoriamente o no) el centro de su angustia. El objetivo general del tratamiento suele verse profundamente afectado en estos casos. Las muertes absurdas más frecuentes pertenecen a tres grupos: 1) inesperadas, 2) deteriorantes o mutilantes y 3 ) aquellas que alteran el orden generacional.

Una de las experiencias más dramáticas que un terapeuta puede tener que afrontar es la experiencia de un paciente que pierde un hijo. A la vuelta de unas vacaciones me en- 
contró con una paciente que había recibido la información médica de que su único hijo padecía de una leucemia. La paciente estaba desgarrada por esta circunstancia y aunque se esforzaba por estar fuerte para enfrentar la situación, se encontraba en un estado desesperante. El trabajo terapéutico se vio totalmente rebasado por esta circunstancia, que pasó a ser el único tema de importancia para la paciente. La tarea tuvo que ser brutalmente redefinida con el fin de ayudarla a fortalecerse todo lo que fuera posible para ayudar en el tratamiento de su hijo y enfrentar la eventualidad más que probable de su muerte. Dos años más tarde su hijo murió y para entonces nuestro trabajo habia sido tan intenso como relativo. La paciente emigró y continuó su vida en otro país donde se esforzó por generar nuevos proyectos. Años después me visitó, transmitiéndome una actitud de profunda gratitud. La paciente sentia que la habia ayudado mucho en ese trance y tenía la sensación de que se había volcado plenamente al cumplimiento de su deber del mejor modo posible. No obstante ello, la experiencia terapéutica habia quedado marcada por la sensación de una limitación fundamental, de la inevitabilidad de un hecho que nos arrojaba en un mundo tremendamente doloroso. Una síntesis razonable de todo ello es que la utilidad de la psicoterapia, como cualquier relación de ayuda, debe ser evaluada dentro de los límites de lo posible.

\section{LA EXPERIENCIA DE LA PROPIA MUERTE}

Cuando avanzamos hacia procesos de cambio profundos, cuando involucramos los procesos nucleares en los objetivos del tratamiento, cuando ponemos en juego la arquitectura básica de las construcciones de identidad del paciente para tratar de responder a su sufrimiento y procurar ayudarlo en busca de alivio, entonces la experiencia de la propia muerte se instala en el centro de la escena y juega un papel primordial en la narrativa del tratamiento. ¿Cómo aparece la experiencia de la propia muerte en la terapia? ¿Cómo interviene en el tratamiento?

La posición que cada persona tiene en la vida es el resultado del interjuego que operan tres parámetros fundamentales: a) el nivel de gratificación de la experiencia corporal; b) el nivel de participación en el esquema de poder de las redes en que se desenvuelve esa experiencia; $c$ ) la peculiar manera en que cada persona se sitúa para enfrentar su propia muerte. Estos tres parámetros se organizan en la conciencia evolutivamente, siguiendo un orden de sucesión que respeta la secuencia enunciada. El primer parámetro se organiza desde el comienzo de la experiencia, el segundo se desarrolla a partir del momento en que el individuo es capaz de organizar construcciones significativas de dominio, y el tercero se hace efectivo alrededor de la adolescencia, cuando la persona debe conformar su construcción de autonomía. Dado que los diferentes parámetros se integran progresivamente, la aparición de cada uno de ellos se articula con los anteriores, resignificándolos. La conformación del tercer parámetro tiene, por lo tanto, el efecto de teñir la totalidad de las operaciones de que sirven los individuos para efectuar el balance autoevaluativo de su desarrollo personal.

A partir de la aparición de este tercer parámetro, todas las experiencias que llevan a cabo los seres humanos están condicionadas por la manera en que definen esta dimensión y la vivencia de la funcionalidad o disfuncionalidad de su representación de la realidad pasa a depender de este esquema. La funcionalidad de una construcción de la realidad a partir de la adolescencia es el producto, en última instancia, del modo en que está sellada esta disposición para enfrentar la propia muerte. Pero ¿qué significa esto de enfrentar la propia muerte?.

\section{La certeza de la muerte}

Los seres humanos aprendemos en el curso de nuestra evolución (habitualmente alrededor de la adolescencia), que el fenómeno de la muerte no es, para nosotros mismos, un hecho contingente, algo que puede o no ocurrir, sino una situación inevitable, que deberemos atravesar necesariamente. Aprendemos, desde ese momento, que la muerte no es solamente un estado que afecta a los fenómenos vivientes, sino algo que se nos impone 
como ineludible. Desde ese momento, la muerte aparece como el prototipo de la experiencia de certidumbre, pues contrariamente a lo que ocurre con las otras circunstancias de la vida, ésta se encuentra inexorablemente ligada a nuestra existencia y habrá de mostrar, tarde o temprano, su certeza definitiva. A partir de entonces, lo único completamente verdadero es la muerte. $O$, en palabras de Maeterlinck: "Tanto en nuestra vida como en nuestro universo hay un solo acontecimiento que cuenta: nuestra muerte". ¿Cómo se lleva a cabo ese aprendizaje?. ¿Qué caminos evolutivos atraviesa?. En primer lugar, influye de manera poderosa el molde cultural en que estamos inmersos. Existen formas culturales que facilitan ese aprendizaje y predisponen a un buen afrontamiento con el tema, mientras que otras proponen diversas estrategias evitativas del problema. Nuestra cultura occidental contemporánea parece propiciar conductas bastante elusivas al respecto. Por el contrario, en la cultura de las comunidades religiosas, asi como la de otros grupos comprometidos con proyectos trascendentes, se suele manifestar una fuerte predisposición a enfrentar el problema. Un buen ejemplo de ello se sintetiza en el saludo que saben intercambiar los habitantes de ciertos centros de retiro, como los monasterios de los frailes cartujos :

"Morir habemos", dice uno de ellos, mientras el otro responde:

"Ya to sabemos".

Ninguna imagen tan viva de esta inmediatez, de esta presencia inexorable pero calma, como la configuración arquitectónica de uno de esos centros de origen y configuración medievales como la Cartuja de Portaceli en Valencia. Todas las celdas que sirven para la clausura están dispuestas en torno a un cuadrado que comunica, hacia el exterior, hacia un abismo montañoso, mientras hacia el interior conduce a un atrio donde se encuentra el cementerio del monasterio. Al salir de sus celdas para asistir a los rezos, sus habitantes se encuentran, por lo tanto, con la presencia del cementerio como espacio que los aguarda.

La otra forma de aprendizaje deriva de la peculiar manera de procesamiento personal que cada individuo ha llevado a cabo a través de su propia experiencia. En relación con este segundo aspecto, la psicoterapia tiene un terreno fundamental de trabajo. En particular, debido a que, en cada modalidad específica de evolucionar la conciencia, podemos encontrar los recursos de cada persona para enfrentar la propia muerte. ¿Qué hitos suelen marcar los tiempos de esa evolución?

Hay un primer tiempo de nuestra vida en que la muerte sólo existe como la muerte de los otros. Algunas personas conocen de esto desde momentos excepcionalmente tempranos. En el caso de niños cuyas madres mueren en el parto, la muerte se registra como pura ausencia. En otros, que asisten a la muerte de padres o hermanos en los primeros momentos de la vida, la muerte se concreta en la forma de una desaparición. Cuando ninguna de esas experiencias límites ocurre, la percepción de la muerte es un registro distanciado en el universo observacional del individuo.

Sólo en un segundo tiempo, la muerte se internaliza como una idea que recorre las fases de desarrollo de la abstracción, desde lo concreto hasta lo simbólico, donde alcanza el carácter de un representante privilegiado. Presencia de una idea que adopta una figura mítica: la calavera errante con una hoz cegadora, la Parca cortando los hilos. Pero alli, la muerte todavia resulta distante, está situada en un tiempo futuro, radicalmente separado del presente por una profunda brecha.

Esta simultaneidad de los tiempos es otra característica de la adolescencia y de los pasos que se dan en ese momento evolutivo en la constitución de la identidad personal. Los adolescentes suelen tener conductas que los asemejan a personajes inmortales: desafían abiertamente peligros que los adultos consideran insalvables, desconocen lo amenazante de situaciones que las personas adultas viven con una importante cuota de temor, sienten que sus proyectos cuentan con todo el tiempo del mundo para llevarse a cabo y suelen sentir que su capacidad corporal los habilita para afrontar intrépidamente todo tipo de exigencias y dificultades. Los adolescentes actúan como si no fueran a morir nunca, pero a diferencia de los niños que todavía no configu- 
raron un esquema de la muerte como algo inevitable, los adolescentes se enfrentan al hecho de que ello habrá de ocurrirles también a ellos. Por lo tanto, son inmortales al mismo tiempo que descubren su inherente mortalidad. $Y$ se vuelven inmortales justamente por ello, por la necesidad de oponer a ese descubrimiento brutal y descarnado, la construcción de una fuerza casi infinita que se le contraponga.

De alli en adelante, el individuo debe responder a ese hallazgo. La vivencia de su propia muerte estará jalonada por distintos momentos, cuya forma y modalidad será infinitamente variable. A la constatación inicial de la inevitabilidad le seguirá un tiempo en que la muerte se convierte en una constante potencial a través de sus representantes cotidianos: pérdidas, frustraciones, paso del tiempo. Más tarde aún, se instala frente a nosotros y su proximidad nos exige recoger lo que hemos ido desplegando a lo largo de nuestra vida. Finalmente, se vuelve inmediata, nos acompaña día a día, se encarna y llegamos a entablar con ella un diálogo permanente.

\section{La psicoterapia y la experiencia de la propia muerte}

Dos son las experiencias más dramáticas que suelen presentarse en la psicoterapia. En primer lugar, la situación de los pacientes que padecen una enfermedad terminal. En segundo lugar, la condición suicida. A continuación, nos centraremos en la primera de estas situaciones. ¿Qué papel le cabe a la psicoterapia en estos casos?. En términos generales se espera que sirva para aliviar la angustia que supone enfrentar ese trance y ayudar al paciente a atravesar el umbral que lo conecta con la muerte. Un tratamiento psicológico deberá colaborar para que la persona pueda despedirse del mejor modo posible de su mundo y experimentar el mejor balance de su gestión en la vida.

En los últimos años se han presentado distintas propuestas para ayudar a estos pacientes. Algunas, como la de Kübler-Ross (1987), contienen una fuerte dosis de religiosidad. Entre los abordajes psicoterapéuticos más habituales se encuentran técnicas de apoyo, terapia familiar y terapia grupal (Levy, 1990). ¿De qué depende la capacidad de los seres humanos para enfrentar adecuadamente su propia muerte?. ¿Cómo puede ayudarlos la psicoterapia cuando es necesario?.

Tenemos dos modos básicos de enfrentar nuestro propia muerte: aceptación o rechazo. Cuando predomina este último, la actitud negadora del individuo se expresará por medio de ideas racionalizadas $u$ omnipotentes. Una vida gobernada por dicha negación requiere superficializar la experiencia y propiciar conductas permanentes de huida. Por el contrario, la capacidad para enfrentar la propia muerte con una actitud de aceptación, está en directa relación con el modo en que cada persona siente que ha podido desplegar su guión y, por lo tanto, que siente haber cumplido con su proyecto personal. Kolakowski (1970) dijo: "Si es verdad que el sentimiento de una vida llena de sentido exige afirmar 10 indudablemente inevitable, entonces la afirmación de la vida es a la vez una afirmación de la muerte".

La decisión de enfrentar la propia muerte es un gesto de la conciencia. La toma de conciencia que acompaña cada momento significa, en alguna medida, morir un poco. Lo que suele ir acompañado de una sensación de temor, aquella misma sensación a la que alude el humanismo de Hamlet. En relación con ello, Kolakowski agrega que el miedo a la muerte tiene dos causas heterogéneas y que con frecuencia, no coinciden entre sí, ni siquiera en sus efectos. Hay un miedo a la muerte concreta que nos protege contra el peligro que amenaza nuestra vida y un miedo a la muerte abstracta, a la muerte en general. La memoria es el pasado hecho consciente. Por ello, el temor a la muerte es el temor a perder el pasado. Adolfo (48), un prestigioso arquitecto, pidió ayuda cuando enfrentaba la fase terminal de un cáncer de colon, del que había sido operado 6 meses antes. La posterior aparición de metástasis volcaron el pronostico hacia un final próximo. Su actitud parecia ser, fugazmente, aceptante de la situación. Pero, casi inmediatamente, se refugiaba en ideas mágicas y en la búsqueda de soluciones casi fantásticas. Dialogando con él encontramos que su vida estaba profundamente quebrada en el plano de los afectos primarios. Su pareja estaba 
destruída desde hacia varios años, a pesar de que seguía viviendo con su esposa, a quien despreciaba y de quien sentia recibir conductas muy destructivas. Con sus hijos sentía una profunda incomunicación, traducida especialmente en la vivencia de no recibir cariño ni cuidado de ellos. Sentía que sólo había triunfado en el nivel laboral pero ello estaba muy alejado de lo que se había propuesto en la vida.

La enorme infelicidad que sentía fue el blanco del trabajo terapéutico que duró apenas tres meses, el tiempo de vida que su cáncer le permitió. Adolfo, un hijo mayor en un contexto familiar muy exigente, era una persona rígida y omnipotente. La sobreexigencia que lo caracterizaba requería proponerle un diálogo sofisticado, con el fin de obtener crédito para las intervenciones terapéuticas. Que se orientaron, de manera sistemática, a proponerle nuevas interpretaciones posibles acerca de su estado, su visión de la enfermedad $y$ de sus posibilidades. Cada sesión creaba un nuevo mundo posible. $Y$ en todas las sesiones, comprobábamos el anhelo que sentía por intentar la reorganización de su guión. Asi, pudo acercarse a sus hijos y establecer un diálogo con ellos que nunca había tenido. A pesar de lo breve del tiempo, pudimos hacer muchas cosas que resultaron beneficiosas para él. Dos años después, un familiar suyo nos contó sus impresiones sobre ese final de su vida, y sobre los últimos momentos en particular. Dichas impresiones reafirmaron nuestra sensación positiva del proceso que habíamos recorrido juntos.

\section{Cómo se construyen los sistemas para enfrentar la propia muerte}

Para enfrentar la propia muerte es necesario, en primer lugar, poder posicionarse frente a ella sin alejarse (actitud fóbica) ni acercarse (actitud desafiante o maníaca) excesivamente, sino interiorizando su presencia a través de las múltiples representaciones que tiene: la muerte de los otros, el paso del tiempo, el agotamiento del deseo. Si logramos esa posición, nuestro trato con ella será igual al balance que hagamos de nuestra realización personal. Si la conciencia de la propia muerte se torna insoslayable desde el momento en que se alcanza el plano de la autonomía personal, la posibilidad de conformar sistemas sólidos para enfrentarse con la muerte, surgirá de la capacidad que el individuo tenga para desarrollar estilos constructivos, primero transitivos y luego perdurables. Si logramos organizar transitivamente nuestra realidad, podremos cumplir un plan que involucre a otro u otros. Nuestro diálogo con la muerte habrá avanzado con ello, pues para acceder a ese espacio de transitividad, habremos sufrido una primera muerte: la de nuestro egocentrismo. Amar es comenzar a morir. De alli que se diga que al amor salva de la muerte, lo que significa que nos justifica en el hecho de haber vivido. Por eso, los amantes experimentan la sensación de que podrian morir en el mismo acto que los reúne.

Si avanzamos por la senda de la transitividad nos sentiremos impulsados a generar obras que traduzcan la germinación de ese encuentro. El fruto de esa unión será la creación de algo que esperamos habrá de perdurar más allá de nosotros. Con ello, aumentamos decididamente nuestra capacidad de enfrentar a la muerte, pues si somos capaces de perdurar, entonces nuestro encuentro con ella no nos hace desaparecer sino que nos transfiguramos en ello. Seremos otro. Una obra supone un acto que implica cierta manera de morir. Por eso decimos que en la gestación de un hijo se muere de alguna manera. La asimetría generacional impone una ley sucesoria al mismo tiempo que garantiza nuestra continuidad. Estar preparados para morir depende de sentir que los actos de nuestra vida se justifican por su capacidad de perpetuarse, por su posibilidad de perdurar en otros. Eso explica por qué las personas que logran desarrollar una vida basada en el cumplimiento efectivo de valores trascendentes (por ejemplo religiosos), suelen tener mejores posibilidades de estar bien situados para enfrentar la muerte.

El trabajo terapéutico con Adolfo se orientó en esa dirección y seguramente, en el hecho de haber encontrado esa postrer comunicación con sus hijos radicó buena parte del alivio 
que experimentó. Esta transformación es posible hasta el último instante de una vida, y por ello, el trabajo con pacientes aún en situaciones desesperadas, merece la pena ser llevado a cabo. Idea que puede leerse maravillosamente relatada en el cuento de Tolstoi, "La muerte de Iván llich".

Del suicidio, experiencia absoluta del morir, y su lugar en la psicoterapia, valdrá la pena ocuparse en otro trabajo. Por ahora, sólo unas palabras para terminar, en los versos de Alejandra Pizarnik.

golpean las sombras negras

las sombras negras

de los muertos

nada sino golpes

$y$ se ha llorado

basta, de querer entender el sufrimiento

nada sino golpes

\section{REFERENCIAS BIBLIOGRÁFICAS}

Aries, Ph. (1983). El hombre ante la muerte. Madrid: Taurus

Hertz, R. (1990). La muerte y la mano derecha. Madrid: Alianza.

Jaspers, K. (1967). Psicología de las concepciones del mundo. Madrid: Gredos.

Kübler-Ross, E. (1989). La muerte: un amanecer. Barcelona: Luciérnaga.

Levinas, E. (1994). Dios, la muerte y el tiempo. Madrid: Cátedra.

Levy, S. M. (1990). Humanizing death: psychotherapy with terminally ill patients. En: P. T. Costa y G. R. VandenBos (Eds.), Psychological Aspects of Serious Iliness. Washington: American Psychological Association.

Thomas, L.V. (1983). Antropología de la muerte. México: Fondo de Cultura Económica.

Yalom , I. (1984): Psicoterapia Existencial. Madrid, Herder. 